\title{
MRI Assisted Stereotactic Biopsy (MAST) in Patients with Elevated Prostate Cancer Risk and Previous Negative Biopsy
}

\author{
Gabriella Ghanem¹, Danny Vesprini, ${ }^{1,2}$, Masoom A Haider ${ }^{3}$, Laurent Milot ${ }^{3}$, Jack Barkin ${ }^{4}$, Petar Erdeljan ${ }^{5}$, Shawn \\ Soon ${ }^{5}$ and Andrew Loblaw ${ }^{1,2,6 *}$ \\ ${ }^{1}$ Odette Cancer Centre, Sunnybrook Health Sciences Centre, Canada
}

${ }^{2}$ Department of Radiation Oncology, University of Toronto, Canada

${ }^{3}$ Department of Medical Imaging, Sunnybrook Health Sciences Centre, University of Toronto, Canada

${ }^{4}$ Division of Urology, Humber River Regional Hospital, Canada

${ }^{5}$ Division of Urology, The Scarborough Hospital, Canada

${ }^{6}$ Institute of Health Policy, Measurement and Evaluation, University of Toronto, Canada

Submission: May 05, 2017; Published: July 07, 2017

*Corresponding author: Andrew Loblaw, Institute of Health Policy, Measurement and Evaluation, University of Toronto, Bayview Avenue, Sunnybrook Health Sciences Center, Toronto, ON Canada, Tel: 416480 4806; Fax: 416480 6002; Email: andrew.loblaw@sunnybrook.ca

\begin{abstract}
Purpose: To report on the Sunnybrook experience of MAST biopsies in patients with one or more previous negative prostate biopsies and an elevated prostate cancer risk - referred to as "Discordant PSA" patients.

Materials and methods: 100 patients with an elevated/rising PSA and at least one previous negative prostate biopsy were included in this study. The University of Texas's PCPTRC was used to articulate the patient's risk of all cancer and G7+ cancer. All 100 patients received an mpMRI. Patients with a G7+ risk of more than $5 \%$ and a nodule on mpMRI were offered a MAST biopsy. Results of the MRI and MAST biopsies were collected and analyzed further.

Results: Overall, 9 out of 24 patients who received a MAST biopsy had clinically significant cancer. Pre-MRI G7+ risk correlated strongly with PI-RADS class (spearman's rank 0.90, $\mathrm{p}=0.037$ ). The calculated positive likelihood ratios for PI-RADS 3-5 were $0,1.2$ and 7.1, respectively (positive likelihood ratio= post-test odds/pre-test odds) [1].
\end{abstract}

Conclusion: MAST biopsies proved to be a useful method in detecting clinically significant prostate cancer in discordant PSA patients with a PI-RADS class 4-5 lesion. They also proved useful in eliminating unnecessary repeat biopsies in discordant PSA patients.

Keywords: Prostate cancer; Magnetic resonance imaging; Stereotactic biopsy

\section{Introduction}

Prostate cancer is the most commonly diagnosed noncutaneous malignancy among men in most western countries [2]. In general, an abnormal serum PSA test and/or a suspicious DRE are the first indicators of prostate cancer and warrant further investigations [3]. A TRUSGB is currently the gold standard for confirming prostate cancer diagnoses. PSA tests are characterized by high sensitivity and low specificity whereas TRUSGB are characterized by their low sensitivity and high specificity [4]. In some cases a TRUSGB will not detect any prostate cancer in a patient with an abnormal DRE and/or rising
PSA despite the patient going on to be diagnosed with the disease on repeat biopsy or clinical progression (i.e., false negative).

A negative TRUSGB will provide some reassurance by lowering the patient's risk of having prostate cancer. However, depending on other predictive factors, the patient may still be at substantial risk which can be articulated by nomograms or online calculators $[4,5]$. We deem these patients "discordant PSA" patients - those with one or more negative prostate biopsies and a persistently elevated prostate cancer risk as determined by PSA and other predictive factors. 
Patients who fall into this category have a few options including: PSA surveillance, repeat TRUSGB, alternative biomarker assay [6], initiating a 5-ARI [7] to elicit a PSA response or multiparametric magnetic resonance imaging \pm targeted biopsy.

TRUSGB is uncomfortable even with the use of periprostatic anesthetic blocks. Of greater concern, however, are the increasing risks of the procedure which include urinary tract infections, hematuria and acute urinary retention [8]. In a populationbased study of Ontario patients who received TRUSGB, Nam et al. [8] reported that the rate of patients readmitted to a hospital 30 days after the procedure increased from $1.0 \%$ in 1996 to $4.1 \%$ in 2005 [8]. The increase in admission was due to infection (bleeding and obstruction admissions were stable). In Alberta in 2012, the incidence had risen to $6.3 \%$ [9]. Given the diminishing yield of repeat TRUSGB and increasing complications, a different approach that may demonstrate a higher positive predictive value warrants investigation.

PCA3 is a non-protein coding RNA that is over expressed in prostate cancer, is measureable in urine, does not correlate with benign prostate volume and is not present in benign prostate diseases. Quantification of PCA3 mRNA levels can predict the outcome of prostatic biopsies with a higher specificity in comparison to PSA [6]. Calculators are available that refine the risk estimates, including the PCA3, but often a persistently elevated risk remains [5]. 5-ARIs have been shown to reduce the risk of low grade prostate cancers in men with discordant PSAs, but do not reduce the risk of clinically significant disease (namely intermediate- and high-grade disease) [7] and may falsely reassure the patient by lowering his PSA even though high grade disease might be present. Post-nadir kinetics can however offer a clue about which patients have underlying highgrade disease [10].

There is increasing interest in the role of mpMRI in the diagnosis of prostate cancer. Typical sequences include T1, T2, Diffusion Weighted imaging with calculation of ADC and DCE. Prostate tumors (particularly in the peripheral zone) are dark on $\mathrm{T} 2$, show bright/early contrast uptake and have low ADC values [11]. Lower ADC values have been associated with higher grade disease [12]. It has been argued that the greatest clinical utility for an MRI is as a triage test to rule out high grade disease so that men can avoid unnecessary repeat biopsies [1]. Additionally, MR imaging has proved a useful imaging technique in discriminating clinically significant cancers in biopsy naïve prostate cancer [13]. A MRI-assisted stereotactic biopsy (MAST) biopsy of the prostate can then be used to confirm the presence or absence of clinically significant disease.

This study reports the Sunnybrook experience of MAST biopsies in patients with discordant PSAs. We will describe the pre-MRI risk of patients referred with discordant PSAs; the results of the MRIs; the proportion of patients who had a MAST biopsy and the results of those biopsies.

\section{Methods}

\section{Patients}

Patients referred to Sunnybrook Health Sciences Centre for consideration of a MAST biopsy were considered for this study. They were referred by their urologists due to one or more negative biopsies and a rising or elevated PSA ("discordant PSA"). During their initial consultation, the patient's risk of prostate cancer was calculated using the University of Texas's PCPTRC. This was done using the patient's age, ethnicity, DRE result, latest PSA (including the free to total ratio), family history, and whether the patient had a previous biopsy.

We included all referred patients who had at least one negative biopsy and a rising or elevated PSA. Patients were excluded if they had a history of prostate cancer oral contraindication to MRI. A convenience sample of one hundred consecutive patients referred between January 2012 and July 2014 were included in this study.

\section{Pre-MRI}

Data was collected from patient charts and electronic patient records and used to calculate the risk of prostate cancer (any grade) and the risk of high grade (Gleason 7 or higher) cancer using the PCPTRC. PSA was adjusted if the patient was on 5-ARIs. Information on whether or not the patient had received a PCA3 test was also collected.

\section{MRI}

MRI was performed on a 3T MR imaging system (Achieva, Philips Healthcare, Best, The Netherlands) without an endorectal coil using a 6-channel cardiac surface coil (SENSE, Philips Healthcare, Best, The Netherlands). Pulse sequence parameters were as follows: axial, sagittal, and coronal T2-weighted (TR $4000 \mathrm{~ms}$, TE $110 \mathrm{~ms}$, field of view $22 \mathrm{~cm}$, matrix $440 \times 425$, number of acquisitions 1 , slice thickness $3 \mathrm{~mm}$, slice spacing $0 \mathrm{~mm}$ ); diffusion-weighted (TR $6178 \mathrm{~ms}$, TE $61 \mathrm{~ms}$, field of view $20 \mathrm{~cm}$, matrix $136 \times 136$, number of acquisitions 3,10 , and $16, \mathrm{~b}$ values $100,400,1000$, slice thickness $3 \mathrm{~mm}$, slice spacing $0 \mathrm{~mm}$ ), and dynamic contrast enhanced imaging (TR $3.9 \mathrm{~ms}$, TE $1.8 \mathrm{~ms}$, field of view $26 \mathrm{~cm}$, number of acquisitions 4 , slice thickness $3 \mathrm{~mm}$, slice spacing $0 \mathrm{~mm}$. Exams were read by one of two UroRadiologist (MAH, LM). The highest Prostate Imaging-Reporting and Data System (PI-RADS) score, and the size and location of nodules were recorded [14].

\section{MAST Biopsy}

Patients were offered biopsies if they had a G7+ risk of more than $5 \%$ and a nodule on MRI. The rationale for this comes from looking at the risk of complication after a TRUSGB - which is 4.1$6.3 \%$ in patients without cancer $[8,9]$. Targeted and $12 \times 12 \mathrm{~mm}$ core systematic MAST biopsies were done using MR/3D ultrasound fusion platform (Eigen, Grass Valley). Some patients who had prostatitis on a previous biopsy had a targeted and 
$6 \times 22 \mathrm{~mm}$ core US-guided transperineal biopsies using cognitive co-registration of MR images. Information on the number of cores taken, the number of cores involved, Gleason sum, total surface area, and percentage Gleason 4/5 involvement was included. Outcomes of patients with nodules who did not go on to have a MAST biopsy were also included.

\section{Analysis}

The primary outcome was the number of patients diagnosed with G7+prostate cancer. We also describe the pre-MRI risk of patients referred with discordant PSAs; the results of the MRIs (by PI-RADS class); the proportion of patients who had a MAST biopsy and the results of those biopsies. Simple descriptive statistics were used to report the results. Spearman's rank coefficient was used to correlate pre-MRI G7+ risk and PI-RADS class. A convenience sample size of 100 was used to get point estimates of frequencies of the outcomes for planning of future trials.

\section{Results}

Table 1: Demographic and Clinical Information of 100 Patients Referred for Discordant PSA and Negative Biopsies based on MAST and MRI Results.

\begin{tabular}{|c|c|c|c|c|c|c|}
\hline & & All Patients & MRI & & MAST & \\
\hline & & & Clear & Nodule & Tumour & Benign \\
\hline Mean Age (Range) & & $64(46-83)$ & $64(46-79)$ & $67(50-83)$ & $67(51-83)$ & $66.4(57-79)$ \\
\hline $\begin{array}{l}\text { Median PSA ng/ml } \\
\text { (Range) }\end{array}$ & & $8.26(1.79-4.80)$ & $8.16(1.79-24.7)$ & $8.6(4.31-54.8)$ & $10.6(5.17-54.8)$ & $7.9(4.31-24.7)$ \\
\hline $\begin{array}{l}\text { Mean neg bx } \\
\text { (Range) }\end{array}$ & & $2.06(1-5)$ & $2.24(1-5)$ & $1.66(1-3)$ & $1.78(1-3)$ & $1.57(1-3)$ \\
\hline \multirow[t]{2}{*}{$\begin{array}{c}\text { Mean PCPTRC risk } \\
\text { \% (Range) }\end{array}$} & All ca & $41.05(12.2-80)$ & $39.1(17-64)$ & $45.93(12.2-80)$ & 55.49 (29.9-75) & $39.66(25-61.7)$ \\
\hline & $\mathrm{G} 7+\mathrm{ca}$ & $18.48(3.9-62.9)$ & $15.74(3.9-46)$ & $25.51(5-62.9)$ & $34.9(5.7-62.9)$ & $17.75(6.3-39.1)$ \\
\hline \multirow[t]{5}{*}{ Gleason Score } & All ca & & & & 9 & \\
\hline & 3,3 & & & & 1 & \\
\hline & 3,4 & & & & 2 & \\
\hline & 4,3 & & & & 5 & \\
\hline & 4,4 & & & & 1 & \\
\hline \multirow[t]{5}{*}{ Treatment } & AS & & & & 1 & \\
\hline & Radiation & & & & 3 & \\
\hline & Surgery (rP) & & & & 3 & \\
\hline & Lost to FU & & & & 1 & \\
\hline & Pending & & & & 1 & \\
\hline
\end{tabular}

Table 2: Results by PI-RADS class.

\begin{tabular}{|c|c|c|c|c|c|c|}
\hline $\begin{array}{c}\text { Max PI-RADS } \\
\text { Class }\end{array}$ & n & Mean G7 + Risk & $\begin{array}{c}\text { Biopsies Done } \\
\text { (\%) }\end{array}$ & $\begin{array}{c}\text { Cancer } \\
\text { Confirmed (\%) }\end{array}$ & G7+ Ca Found & $\begin{array}{c}\text { Positive } \\
\text { Likelihood Ratio }\end{array}$ \\
\hline 1 & 15 & $12.3 \%$ & 0 & & & \\
\hline 2 & 49 & $15.9 \%$ & 0 & & & \\
\hline 3 & 19 & $20.4 \%$ & $9(47.4 \%)$ & 0 & & $1(100 \%)$ \\
\hline 4 & 6 & $17.0 \%$ & $5 *(83.3 \%)$ & $1(20 \%)$ & 1.2 \\
\hline 5 & 11 & $36.0 \%$ & $10^{*}(90.9 \%)$ & $9(90 \%)$ & $8(88.9 \%)$ & 7.1 \\
\hline
\end{tabular}

One patient refused biopsies

The clinical and demographic information of the cohort is summarized in Table 1. Of the 100 patients who received an MRI, the mean age of the cohort was 64 years (range $46-83$ years) and their median PSA was $8.26 \mathrm{ng} / \mathrm{ml}$ (range $1.79-54.8 \mathrm{ng} / \mathrm{ml}$ ). These men had a median of 2 previous negative biopsies (range 1-5). Four had PCA3 testing. The mean G7+ risk was 25.5\% (range $3.9 \%-62.9 \%)$. 
The distribution of PI-RADS score in patients who underwent an MRI is summarized in Table 2. Sixty-four patients were PIRADS class 1-2. None were offered biopsy. It should be noted that one of these patients with a PI-RADS class 2 had an MRI nodule but his pre-MRI G7+ risk was $7 \%$ and he had 2 previous negative biopsies. After 1.5 years of follow-up his PSA remained stable and he was discharged to his community Urologist.

Pre-MRI G7+ risk correlated strongly with PI-RADS class (Table 2), Spearman's rank 0.90, p=0.037). Of the 19 patients that were PI-RADS class 3, 9 (47\%) had biopsies and all were negative (Table 2). Eleven of these 19 patients had MRI nodules ( 6 had a negative biopsy, 5 were observed). Of the 5 observed, 3 subsequently had repeat MRIs, one of which was PI-RADS class 4 . He had rapidly progressing dementia so it was elected to observe him clinically.

One of the 5 patients (20\%) with PI-RADS class 4 lesions had cancer (Gleason 8); 9 of 10 patients (90\%) of patients with PI-RADS class 5 lesions had cancer (1 Gleason 6, 8 Gleason 7). Overall 9 of the 24 patients (37.5\%) who received a MAST biopsy were found to have G7+ prostate cancer. The calculated positive likelihood ratios for PI-RADS 3-5 were 0, 1.2 and 7.1, respectively (positive likelihood ratios=post-test odds/pre-test odds) [1].

\section{Discussion}

In this study we set out to identify how many cases of clinically significant prostate cancer would be identified in patients with a discordant PSA and one or more previous negative biopsies after an MRI and MAST biopsy. Overall we identified 10 prostate cancers in our 100 patients, 9 of them being clinically significant. Importantly, however, we were able to avoid repeat biopsies in $76 \%$ of patients. Given the discomfort and risk of complications from TRUSGB $[8,9]$ not to mention that men had a median of 2 previous TRUSGB, we believe this is beneficial for patients. Since our study was initiated, the UK's NICE prostate guidelines recommend considering the use of multi-parametric MRI for patients with a discordant PSA [16].

Observing patients with PI-RADS class 1-2 MRIs seems to be advisable. One long-term study from Itatani et al. [17] followed 193 patients with negative initial TRUS-guided biopsy findings and PI-RADS class 1-2 scans for 5 years after their initial MRI. Only patients with negative findings by digital rectal examination, MRI, and repeat biopsy and no increase in PSA at 5-year followup were defined as "clinically negative". The negative predicted value for significant prostate cancer was $89.6 \%$ [17].

Although there were only 9 (of 19) patients in our study with PI-RADS class 3 lesions who received biopsies, all of these patients had negative biopsies suggesting that biopsying these men held limited value. Similarly, when Abd-Alazeez and colleagues looked at PI-RADS 3-5 classes (using systematic transperineal mapping biopsy, minimum 20 cores, as the gold standard) the chance of finding G7+ disease was $3 / 35$ (8.6\%),
9/23 (39.1\%) and 8/11 (72.7\%), respectively [13]. We would recommend that more data should be collected but that following patients clinically like Itatani et al. [17] did would seem to offer a favourable balance between the harms of futile investigation versus missing clinically significant disease.

One limitation of this study is the lack of follow up in patients with a nodule and PI-RADS of 3. Of the 7 patients with nodules who were not biopsied, 3 went on to have repeat MRIs due to rising PSAs with one having an upgrade to PI-RADS class 4 on repeat MRI. Follow up has the potential to identify the presence of clinically significant cancer in these patients. Our study was smaller than other studies investigating this question $[13,16,18]$, but most of these are not large enough since the chance of having PI-RADS class 4-5 is low (only 17\% in our study). Larger studies, particularly with prospective clinical follow-up for patients with PI-RADS class 1-3, is recommended to improve the confidence of the likelihood ratio calculations.

In this era of increased cost-consciousness, one might be critical that 100 MRIs only found 9 cases of cancer in patients with a discordant PSA and one or more previous negative biopsies. Of greater concern would be the routine use of mpMRI in patients who are biopsy naïve. A study by de Rooji et al. [19] found that the MRI strategy is initially more expensive but these costs are compensated for by reducing treatment costs (in the Netherlands) and avoiding the potential side effects of unnecessary biopsies. As the treatment and MRI reimbursement rates factor heavily into these analyses, one would have to use regional data to determine if this strategy is cost-effective in one's local area. We believe currently there is insufficient evidence to offer MRI screening for prostate cancer using public healthcare resources.

\section{Conclusion}

In patients with a previous negative prostate biopsy and elevated cancer risk, mpMRI with stereotactic biopsis for those with PI-RADS class 4-5 lesions is useful to detect clinically significant cancers without subjecting patients to unnecessary biopsies. More work is needed to identify the appropriate management strategy for these who have PI-RADS class 3 lesions and the cost-effectiveness of this strategy.

\section{References}

1. Nam RK, Kattan MWN, Chin JL, Trachtenberg J, Singal R, et al. (2011) Prospective multi-institutional study evaluating the performance of prostate cancer risk calculators. J Clin Urol 29(22): 2959-2964.

2. Jemal A, Bray F, Center MM, Ward E, Forman D (2011) Global cancer statistics. CA: A Cancer Journal for Clinicians 61(2): 69-90.

3. Fleshner NE, O'Sullivan M, Fair WR (1997) Prevalence and Predictors of a Positive Repeat Transrectal Ultrasound Guided Needle Biopsy of the Prostate. J Urol 158(2): 505-509.

4. Pondman KM, Futterer JJ, ten Haken B, Schultze Kool LJ, Witjes JA, et al. (2008) MR-Guided Biopsy of the Prostate: An Overview of Techniques and a Systematic Review. Eur Urol 54(3): 517-527. 
5. University of Texas Health Sciences Centre (2014) Individualized Risk Assessment of Prostate Cancer. San Antonio, USA.

6. Hu B, Yang H, Yang H (2014) Diagnostic value of urine prostate cancer antigen 3 test using a cutoff value of $35 \mu \mathrm{g} / \mathrm{L}$ in patients with prostate cancer. Tumor Biology 35(9): 8573-8580.

7. Andriole GL, Bostwick D, Brawley OW, Gomella L, Marberger M, et al. (2011) The effect of dutasteride on the usefulness of prostate specific antigen for the diagnosis of high grade and clinically relevant prostate cancer in men with a previous negative biopsy: results from the REDUCE study. J Urol 185(1): 126-131.

8. Nam RK, Saskin R, Lee Y, Liu Y, Law C, et al. (2013) Increasing hospital admission rates for urological complications after transrectal ultrasound guided prostate biopsy. J Urol 183(3): 968-968.

9. Rudzinski JK, Kawakami J (2014) Incidence of infectious complications following transrectal ultrasound-guided prostate biopsy in Calgary, Alberta, Canada: A retrospective population-based analysis. Can Urol Assoc J 8(5-6): E301-305.

10. Marberger M, Freedland SJ, Andriole GL, Emberton M, Pettaway C, et al. (2012) Usefulness of prostate-specific antigen (PSA) rise as a marker of prostate cancer in men treated with dutasteride: lessons from the REDUCE study. BJU Int 109(8): 1162-1169.

11. Hambrock T, Somford DM, Huisman HJ, van Oort IM, Witjes JA, et al (2011) Relationship between apparent diffusion coefficients at 3.0 T MR imaging and Gleason grade in peripheral zone prostate cancer. Radiology 259(2): 453-461.

12. Barentsz JO, Richenberg J, Clements R, Choyke P, Verma S, et al. (2012) ESUR prostate MR guidelines 2012. European radiology. Apr 22(4): 746-757.
13. Abd-Alazeez M, Kirkham A, Ahmed HU, Arya M, Anastasiadis E, et al. (2014) Performance of multiparametric MRI in men at risk of prostate cancer before the first biopsy: a paired validating cohort study using template prostate mapping biopsies as the reference standard. Prostate Cancer and Prostatic Dis 17(1): 40-46.

14. Barentsz JO, Richenberg J, Clements R, Choyke P, Verma S, et al. (2012) ESUR prostate MR guidelines 2012. Eur radiol 22(4): 746-757.

15. Deeks JJ, Altman DG (2004) Diagnostic tests 4: likelihood ratios. Br Med J 329(7458): 168-169.

16. National Institute for Heath and Care Excellence (2004) Prostate Cancer: Diagnosis and Treatment. UK.

17. Itatani R, Namimoto T, Atsuji S, Katahira K, Morishita S, et al. (2014) Negative predictive value of multiparametric MRI for prostate cancer detection: Outcome of 5-year follow-up in men with negative findings on initial MRI studies. European Journal of Radiology 83(10): 17401745 .

18. Hoeks CM, Schouten MG, Bomers JG, Hoogendoorn SP, Hulsbergen-van de Kaa CA, et al. (2012) Prospective assessment of prostate biopsy in men with increased prostate specific antigen and repeated, negative, random, systematic, transrectal ultrasound biopsies: detection of clinically significant prostate cancers. Eur Urol 62(5): 902-909.

19. de Rooij M, Crienen S, Witjes JA, Barentsz JO, Rovers MM, et al. (2014) Cost-effectiveness of Magnetic Resonance (MR) Imaging and MRguided Targeted Biopsy Versus Systematic Transrectal UltrasoundGuided Biopsy in Diagnosing Prostate Cancer: A Modelling Study from a Health Care Perspective. Eur Urol 66(3): 430-436.

\section{Your next submission with Juniper Publishers} will reach you the below assets

- Quality Editorial service

- Swift Peer Review

- Reprints availability

- E-prints Service

- Manuscript Podcast for convenient understanding

- Global attainment for your research

- Manuscript accessibility in different formats

( Pdf, E-pub, Full Text, Audio)

- Unceasing customer service

Track the below URL for one-step submission https://juniperpublishers.com/online-submission.php 